\title{
Enumerating the Morphology of Non-degenerate Darboux Cyclides
}

\author{
Mingyang Zhao ${ }^{\mathrm{a}, \mathrm{b}}$, Xiaohong Jia ${ }^{\mathrm{a}, \mathrm{b}, *}$, Changhe $\mathrm{Tu}^{\mathrm{c}}$, Bernard Mourrain ${ }^{\mathrm{d}}$, Wenping Wang ${ }^{\mathrm{e}}$ \\ ${ }^{a}$ KLMM, NCMIS, Academy of Mathematics and Systems Science, Chinese Academy of Sciences \\ ${ }^{b}$ University of Chinese Academy of Sciences \\ ${ }^{c}$ School of Computer Science and Technology, Shandong University \\ ${ }^{d}$ GALAAD, INRIA Mditerrane \\ ${ }^{e}$ Department of Computer Science, University of Hongkong
}

\begin{abstract}
We provide an enumeration of all possible morphologies of non-degenerate Darboux cyclides. Based on the fact that every Darboux cyclide in $\mathbb{R}^{3}$ is the stereographic projection of the intersection surface of a sphere and a quadric in $\mathbb{R}^{4}$, we transform the enumeration problem of morphologies of Darboux cyclides to the enumeration of the algebraic sequences that characterize the intersection of a sphere and a quadric in $\mathbb{R}^{4}$.
\end{abstract}

Keywords: Darboux cyclides, stereographic projection, Segre characteristic, index sequences, morphologies

\section{Introduction}

Cyclides are originally defined by Dupin (1822), hence since called Dupin cyclides, as algebraic surfaces of degree at most four whose lines of curvatures are all circles. There is a long history of people exploring the geometric properties of Dupin cyclides as well as their applications in geometric modeling, such as serving as blending surfaces and boundary representations (Pratt, 1990, 1995).

Darboux cyclides, as a superset of Dupin cyclides and quadrics, are mainly investigated by Kummer (1865) and Darboux (1880). It is proved that almost every Darboux cyclide contains from two to six real circles through almost every point (Blum, 1980, Coolidge, 1916; Takeuchi et al., 2000), and there is a recent conclusion stating that a surface which carries three families of circles is a Darboux cyclide (Lubbes, 2013). Further explorations on these families of circles on Darboux cyclides can be found in Franquiz et al. (2006) and Pottmann et al. (2012). This circular arc structure of Darboux cyclides attracts the attention of geometric modeling community in applying them to contemporary freeform architecture (Bo et al., 2011, Pottmann et al., 2007, 2008, 2012). However, compared with Dupin cyclides, although with more freedom in the shape and structure, the theoretical investigations of Darboux cyclides in the literature are relatively rare. It is proved in Takeuchi et al. (2000) that a nonsingular Darboux cyclide is topologically a torus, a sphere or two spheres, and that a singular

\footnotetext{
* Corresponding author

Email address: xhjia@amss.ac.cn (Xiaohong Jia)
} 
one is conformally equivalent to a quadric. However, there still lacks an finer enumeration on more detailed morphologies of Darboux cyclides, which is crucial to predicting the local feature in modeling.

Coolidge (1916) has proved that a Darboux cyclide is the stereographic projection of the intersection of a sphere and another quadric in $\mathbb{R}^{4}$, which casts light on our exploration of Darboux cyclides by transiting to the intersection of two quadrics (brief as QSI) in $\mathbb{R}^{4}$. There is rich literature on computation, classification as well as morphology enumeration of the intersection of two quadrics in 3-dimensional space. See Levin (1976, 1979); Miller and Goldman (1995); Wang et al. (2002, 2003); Dupont et al. (2003, 2008a b c); Mourrain et al.(2005); Lazard et al. (2006) for algorithms of computing the exact parametrization of QSIs, and Bromwich (1906); Wang et al. (2003); Tu et al. (2002, 2009) for algebraic approaches to enumerating and classifying the morphologies of QSIs.

We provide a finer enumeration of morphologies of Darboux cyclides, which takes both the topological and algebraic properties of the Darboux cyclides into account. The main result is shown in Theorem 1. which is further merged into 14 non-equivalent morphologies in Table 3 . Besides, Table 4 gives a brief description of all the non-equivalent morphologies. Our enumeration of all possible morphologies of Darboux cyclides is performed by going through all valid QSIs morphologies in $\mathbb{R}^{4}$, inspired by the fact that stereographic projections bridge between Darboux cyclides in $\mathbb{R}^{3}$ and QSIs in $\mathbb{R}^{4}$. The enumeration of all QSIs morphologies is achieved through the enumeration of Segre characteristics and index sequences of the quadric pencil, which generalizes the result of $\mathrm{Tu}$ et al. $(2009)$ from $\mathbb{P R}^{3}$, the $3 \mathrm{D}$ real projective space, to $\mathbb{P R}^{4}$, the $4 \mathrm{D}$ real projective space. Different affine realizations of QSIs

from $\mathbb{P R}^{4}$ to $\mathbb{R}^{4}$ are then considered: realizing the required sphere in the pencil helps define the plane at infinity, and the choices of the other four axes define the stereographic projection center.

The remainder of the paper is organized as follows. In Section 2, we review the previous work about cyclides and QSIs; in Section 3, we present necessary preliminary knowledge on Darboux cyclides, stereographic projections, as well as algebraic characterizations of QSIs; in Section 4 we give an enumeration of the morphologies of Darboux cyclides by going through all suitable QSIs, where detailed proofs are provided. We draw our conclusions in Section 5.

\section{Related Work}

Cyclides. Dupin Cyclides are first defined by the French mathematician Dupin, as the envelopes of all the spheres with possibly distinct radii touching three given fixed spheres (Dupin, 1822). There is plenty of theoretical work exploring the algebraic and geometric properties of Dupin cyclides, see Maxwell (1868); Casey (1870); Cayley (1873) for early theoretical explorations. Cyclide patches are first used in free-form modeling in the early 1980s, see Martin et al. (1982); McLean (1985) for constructions of cyclide patches; a conversion from cyclide patches to rational biquadratic Bézier forms is given by Foufou et al. (2005). Cyclides have fruitful applications in solid geometric modeling due to their lowdegree in both algebraic and parametric representation, intuitive shape parameters, as well as flexibility in free-form surfaces design, see Chandru et al. (1989); Pratt (1990); Allen and Dutta (1997); Foufou and Garnier (2004); Druoton et al. (2014).

Darboux cyclides are a generalization of Dupin cyclides, and have a long history in classical geometry (Coolidge, 1916; Darboux, 1880). Takeuchi et al. (2000) discover that Darboux cyclides can carry up to six families of real circles. Krasauskas and Zubè (2014) study Darboux cyclides using the language of geometric algebra. Recently, Darboux cyclides attract the attention of the geometry modeling community by their circular arc structure. Bo et al. (2011) first apply Darboux cyclides in architecture geometry, where cyclide structures show their power in freeform modeling. Motivated by potential applications of Darboux cyclides in architecture, Pottmann et al. (2012) propose computational tools 
for identification of circle families on a given cyclide, and provide a complete classification of the hexagonal webs on Darboux cyclides.

QSIs. The computation, classification as well as morphologies determination of QSIs is a classic problem both in algebraic geometry and in geometric modeling. Many works target at computing the exact parametrization of the QSIs, while some others focus on morphologies classifications of the QSIs using algebraic tools. Levin (1976, 1979) proposes a parametric algorithm to compute the QSIs in $\mathbb{R}^{3}$ based on the observation that there is a ruled quadric in the pencil of any two distinct quadrics in $\mathbb{P R}^{3}$. Segre (1884) classify pencils of quadrics by Segre characteristic in arbitrary dimensions. Pieter Belmans further points out that the classification of pencils of quadrics in $\mathbb{P R}^{4}$ is equivalent to the classification of Segre quartic surfaces (Belmans, 2016). Wilf and Manor combine Levin's approach with the Segre characteristic to describe the shape and structure of QSIs (Wilf and Manor, 1993). Wang et al. (2003) provide an enhanced version of Levin's method that is able to classify the QSIs and compute a rational parametrization of one QSI when the QSI is a singular curve. Dupont et al. (2003, 2008a b c) give an exact arithmetic to parameterize QSIs in $\mathbb{R}^{3}$, which is near-optimal in the sense that the number of distinct square roots appearing in the coefficients of these parameterized polynomial functions is minimal. Wang et al. (2002) present an algebraic method for classifying and parameterizing the intersection curve of two quadric surfaces, which is based on the observation that the intersection curve of two quadrics is birationally related to a plane cubic curve. Tu et al. $(2002,2009)$ present a complete classification of the QSIs in $\mathbb{P R}^{3}$ using Segre characteristics and index sequences, and an efficient algorithm of determining the morphologies of QSIs is provided, which is mainly based on signature sequence computations. Jia et al. (2016) propose an algorithm of computing the QSI variations for a pair of moving quadrics.

\section{Preliminaries}

\subsection{Morphology}

The morphologies of Darboux cyclides in $\mathbb{R}^{3}$ in this paper is a finer classification than homeomorphism. We take both topological and algebraic properties of the cyclides into account. If two Darboux cyclides are homeomorphic to each other, we further consider the existence and type (cusp, crunode, acnode) of the real singularities, and the separability of the surface in $\mathbb{R}^{3}$ (see Note 1 below). If two cyclides show the same properties in all these factors, and there exists a continuous deformation that sends one cyclide to the other without changing the singularity existence and type (see Note 2 below), we say that the two cyclides have the equivalent morphologies; otherwise they are said to have non-equivalent morphologies, see Fig. 1 for example.
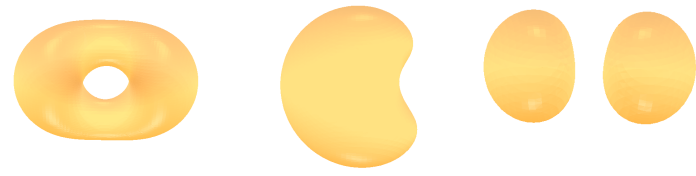

(a) Different topologies: torus, sphere, two spheres
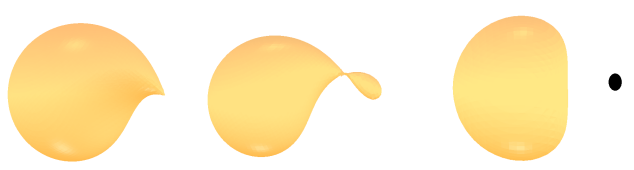

(b) Different types of singularities: cusp, crunode, acnode

Figure 1: Non-equivalent morphologies of Darboux cyclides in terms of (a) topology and (b) type of singularities. 
Note 1. We define the separability of a Darboux cyclide as follows. If a Darboux cyclide $\mathcal{D}$ can be written as $\mathcal{D}=\mathcal{D}_{1} \cup \mathcal{D}_{2}$, where either

1. $\mathcal{D}_{1} \cap \mathcal{D}_{2}=\emptyset$ (case I) or

2. $\mathcal{D}_{1} \cap \mathcal{D}_{2}=\mathbf{Q} \in \mathbb{R}^{3}$, with $\mathbf{Q}$ being a crunode of the surface (case II),

and if there exists a plane $\Pi: L(x, y, z)=0$ such that

1. $\mathcal{D}_{1}=\{x \in \mathcal{D} \mid f(x)>0\}, \quad \mathcal{D}_{2}=\{x \in \mathcal{D} \mid f(x)<0\}$ for case I, and

2. $\mathcal{D}_{1}=\{x \in \mathcal{D} \mid f(x) \geq 0\}, \quad \mathcal{D}_{2}=\{x \in \mathcal{D} \mid f(x) \leq 0\}$ for case II,

then the cyclide is said to be separable; otherwise it is non-separable. See Fig. 2 (a) for case I and (b) for case II.

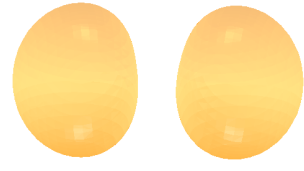

(a) Case I: the left is separable, the right is nonseparable.

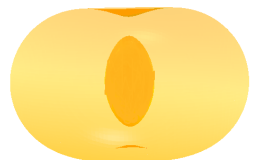

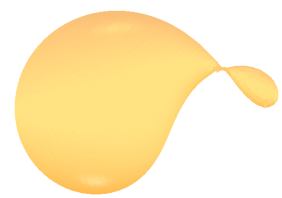

(b) Case II: the left is separable, the right is nonseparable.

Figure 2: Examples of non-equivalent morphologies of Darboux cyclides in terms of separability.

\section{Note 2.}

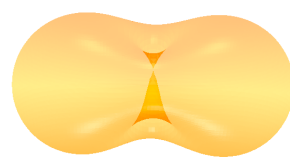

(a) Surfaces with a crunode. To deform the left to the right, the surface needs to be cut at the crunode.
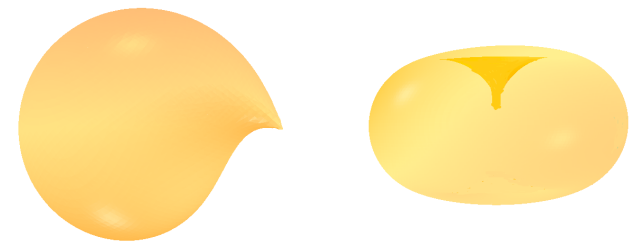

(b) Surfaces with a cusp. To deform the left to the right, there is a instant when the cusp disappears.

Figure 3: Examples of non-equivalent morphologies with the same topology, singularity number and type, separability, but one can not be continuously deformed to the other without changing the existence or type of the singularity.

Subject to the above rules, we derived the following main result of the paper. Detailed analysis will be shown in the later context.

Theorem 1. There are in total 14 non-equivalent morphologies of Darboux cuclides, listed in Table 3.

\subsection{Darboux cyclides and stereographic projection}

A Darboux cyclide in $\mathbb{R}^{3}$ is a quartic algebraic surface with the equation

$$
D(x, y, z):=\lambda\left(x^{2}+y^{2}+z^{2}\right)^{2}+\left(x^{2}+y^{2}+z^{2}\right) L(x, y, z)+Q(x, y, z)=0,
$$


where $\lambda$ is a constant, and $Q(x, y, z)$ and $L(x, y, z)$ are a quadratic and a linear polynomial in $\mathbb{R}[x, y, z]$ (Pottmann et al. 2012). We say that the Darboux cyclide is non-degenerate, if $\lambda \neq 0$ and $D$ is irreducible; otherwise it is degenerate. In this paper we focus only on non-degenerate Darboux cyclides, that is, the cyclide is strictly a degree four algebraic surface. Hence, quadrics and cubics which are special cases for Darboux cyclides are excluded in our analysis.

A stereographic projection with the projection center $\mathbf{O}=(0,0,0,1)$ sends a point $\overline{\mathbf{x}}=\left(x_{1}, x_{2}, x_{3}, x_{4}\right) \in \mathbb{R}^{4}$ on the sphere $\Sigma: x_{1}^{2}+x_{2}^{2}+x_{3}^{2}+x_{4}^{2}=1$ to a point $\mathbf{x}=(x, y, z) \in \mathbb{R}^{3}$ via the map:

$$
\mathbf{x}=(x, y, z)=\frac{1}{1-x_{4}}\left(x_{1}, x_{2}, x_{3}\right)
$$

where the projection center $\mathbf{O}$ is mapped to the ideal point $\infty$, and conversely,

$$
\overline{\mathbf{x}}=\left(x_{1}, x_{2}, x_{3}, x_{4}\right)=\frac{1}{\mathbf{x}^{2}+1}\left(2 x, 2 y, 2 z, \mathbf{x}^{2}-1\right) .
$$

The corresponding points $\overline{\mathbf{x}}$ and $\mathbf{x}$ lie on a straight line through the projection center $\mathbf{O}$.

Lemma 1. Coolidge, 1916) A Darboux cyclide $D \in \mathbb{R}^{3}$ is the stereographic projection of a surface $\bar{D}$ in the unit sphere $\Sigma \in \mathbb{R}^{4}$ which is the intersection of $\Sigma$ with another quadric $\Gamma \in \mathbb{R}^{4}$.

The following derivation of the transformation between Darboux cyclides and QSIs shown in Pottman. n et al. (2012) will be applied to our later analysis.

\section{Cyclide $\rightarrow$ QSI:}

Write the quadratic part in (1) as $Q(\mathbf{x})=q_{2}(\mathbf{x})+q_{1}(\mathbf{x})+q_{0}$, where $\mathbf{x}=(x, y, z)$ and $q_{i}$ is the degree $i$ homogeneous part of $Q$. Substituting (2) into (1) and considering $\Sigma: x_{1}^{2}+x_{2}^{2}+x_{3}^{2}+x_{4}^{2}=1$ yield

$$
\Gamma: \lambda\left(1+x_{4}\right)^{2}+\left(1+x_{4}\right) L\left(x_{1}, x_{2}, x_{3}\right)+q_{2}\left(x_{1}, x_{2}, x_{3}\right)+q_{1}\left(x_{1}, x_{2}, x_{3}\right)\left(1-x_{4}\right)+q_{0}\left(1-x_{4}\right)^{2}=0,
$$

which is a quadric in $\mathbb{R}^{4}$. The QSI is the intersection of the sphere $\Sigma$ and the quadric $\Gamma$.

$$
\text { QSI } \rightarrow \text { Cyclide: }
$$

Conversely, a quadric $\Gamma$ in $\mathbb{R}^{4}$ can be written as

$$
\Gamma: q_{2}\left(x_{1}, x_{2}, x_{3}\right)+q_{1}\left(x_{1}, x_{2}, x_{3}\right) x_{4}+q_{0} x_{4}^{2}+r_{1}\left(x_{1}, x_{2}, x_{3}\right)+r_{0} x_{4}+c_{0}=0,
$$

where $q_{2},\left\{q_{1}, r_{1}\right\}$ and $\left\{q_{0}, r_{0}, c_{0}\right\}$ are the quadratic, linear and constant parts, respectively. Substituting (3) to (5) gives

$$
\left(q_{0}+r_{0}+c_{0}\right)\left(x^{2}+y^{2}+z^{2}\right)^{2}+2\left(x^{2}+y^{2}+z^{2}\right)\left(q_{1}(x, y, z)+r_{1}(x, y, z)+c_{0}-q_{0}\right)+Q(x, y, z)=0,
$$

where

$$
Q(x, y, z)=4 q_{2}(x, y, z)+2\left(r_{1}(x, y, z)-q_{1}(x, y, z)\right)+q_{0}-r_{0}+c_{0} .
$$

Obviously, Equation (6) represents a Darboux cyclide.

The above derivation also suggests

Lemma 2. $\lambda \neq 0$ in Equation (11) if and only if the quadric $\Gamma$ for the QSI does not pass through the stereographic projection center $\mathbf{O}$. 
Let $\mathcal{A}: X^{T} A X=0$ and $\mathcal{B}: X^{T} B X=0$ be two quadrics, where $X=(x, y, z, u, v) \in \mathbb{P R}^{4}$ (4D real projective space) is a homogenous coordinate for $\left(\frac{x}{v}, \frac{y}{v}, \frac{z}{v}, \frac{u}{v}\right) \in \mathbb{R}^{4}$ and $A, B$ are $5 \times 5$ real symmetric matrices. A quadric pencil given by $\mathcal{A}$ and $\mathcal{B}$ is represented as $\lambda A-B$, where $\lambda \in \mathbb{P R}^{1}$ is a constant.

Note that the QSI of any two quadrics in the pencil $\lambda A-B$ is exactly the same as the QSI of $\mathcal{A}$ and $\mathcal{B}$. Therefore, when considering the QSI, we can always assume that $A$ is non-singular; otherwise we can replace $A$ by a nonsigular quadric $A^{\prime}$ in the pencil. Note that there are always nonsingular quadrics if $\operatorname{det}(\lambda A-B)$ does not vanish identically (Tu et al., 2009).

We next review Jordan Forms and Quadric Pair Canonical Forms in Uhlig (1976).

\subsubsection{Jordan forms and Segre characteristics}

Definition 3.1. A $k \times k$ square matrix of the form

$$
M=\left(\begin{array}{cccc}
\lambda & e & & \\
& \cdot & \cdot & \\
& & \cdot & e \\
& & & \lambda
\end{array}\right)
$$

is called a Jordan block of type $I$ associated with $\lambda$ if $\lambda \in \mathbb{R}$ and $e=1$ for $k \geq 2$ or $M=(\lambda)$ with $\lambda \in \mathbb{R}$ for $k=1 ; M$ is called a Jordan block of type $I I$ with respect to complex conjugated values $a \pm i b$ if

$$
\lambda=\left(\begin{array}{cc}
a & -b \\
b & a
\end{array}\right) \mathrm{a}, \mathrm{b} \in \mathbb{R}, \quad b \neq 0 \text { and } e=\left(\begin{array}{cc}
1 & 0 \\
0 & 1
\end{array}\right),
$$

for $k \geq 4$ or

$$
M=\left(\begin{array}{cc}
a & -b \\
b & a
\end{array}\right)
$$

for $k=2$, with $a, b \in \mathbb{R}, b \neq 0$.

Lemma 3. For any matrix $A \in \mathbb{R}^{k \times k}$, there exists a Jordan normal form matrix $J$

$$
\left(\begin{array}{cccc}
C\left(\lambda_{1}\right) & & & \\
& C\left(\lambda_{2}\right) & & \\
& & \ddots & \\
& & & C\left(\lambda_{k}\right)
\end{array}\right)
$$

congruent to $A$, where

$$
C\left(\lambda_{i}\right)=\left(\begin{array}{cccc}
J_{1}^{i} & & & \\
& J_{2}^{i} & & \\
& & \ddots & \\
& & & J_{k_{i}}^{i}
\end{array}\right)
$$

is called the full Jordan chain associated with the eigenvalue $\lambda_{i}, i=1, \cdots, k$, and $J_{1}^{i}, \cdots, J_{k_{i}}^{i}$ are all Jordan blocks (of type $I$ or $I I$ ), associated with the same eigenvalue $\lambda_{i}$ of the matrix $A$. The Jordan normal form is unique up to permutations of the Jordan blocks.

Definition 3.2. The Segre characteristic of the quadric pencil $\lambda A-B$ is the integer chain of orders of the blocks in the Jordan normal form of the matrix $A^{-1} B$, with those integers corresponding to 
blocks containing the same eigenvalue bracketed together, and the number of distinct real eigenvalues of the matrix $A^{-1} B$ as the subscript. For example, if the Jordan form of the matrix $A^{-1} B$ is

$$
\left(\begin{array}{ccccc}
\alpha & 1 & & & \\
& \alpha & & & \\
& & \alpha & & \\
& & & \beta & \\
& & & & \beta
\end{array}\right) \text {, }
$$

where $\alpha$ and $\beta$ are real numbers, the Segre characteristic of the quadric pencil $\lambda A-B$ is $[(21)(11)]_{2}$. In this example, we also say that the Segre characteristic of $\lambda A-B$ associated with the root $\alpha$ is [21], and associated with the $\operatorname{root} \beta$ is [11].

\subsubsection{Quadric pair canonical form}

Lemma 4. (Quadric Pair Canonical Form). Let $A$ and $B$ be a nonsingular pair of real symmetric matrices of size $n$. Suppose that $A^{-1} B$ has real Jordan normal form $\operatorname{diag}\left(J_{1}, \ldots, J_{r}, J_{r+1}, \ldots, J_{m}\right)$, where $J_{1} \ldots, J_{r}$ are Jordan blocks of type $I$ corresponding to the real eigenvalues of $A^{-1} B$ and $J_{r+1}, \ldots, J_{m}$ are Jordan blocks of type $I I$ corresponding to the complex eigenvalues of $A^{-1} B$. Then $A$ and $B$ are simultaneously congruent by a real congruent transformation to

$$
\bar{A}=\operatorname{diag}\left(\varepsilon_{1} E_{1}, \ldots, \varepsilon_{r} E_{r}, E_{r+1}, \ldots, E_{m}\right)
$$

and

$$
\bar{B}=\operatorname{diag}\left(\varepsilon_{1} E_{1} J_{1}, \ldots, \varepsilon_{r} E_{r} J_{r}, E_{r+1} J_{r+1}, \ldots, E_{m} J_{m}\right),
$$

respectively, where $\varepsilon_{i}= \pm 1$ and the $E_{i}$ are of the form

$$
\left(\begin{array}{llll}
0 & \cdot & 0 & 1 \\
\cdot & \cdot & 1 & \cdot \\
\cdot & 1 & \cdot & \cdot \\
1 & 0 & \cdot & 0
\end{array}\right)
$$

of the same size as $J_{i}, i=1,2, \ldots, m$. The signs of $\varepsilon_{i}$ are unique (up to permutations) for each set of indices $i$ that are associated with a set of identical Jordan blocks $J_{i}$ of type $I$.

Since the two canonical quadratic forms are projectively equivalent to the original two quadratic forms, Tu et al. (2009) show the following result.

Lemma 5. The QSI of two quadrics $\mathcal{A}: X^{T} A X=0$ and $\mathcal{B}: X^{T} B X=0$ in $\mathbb{P R}^{4}$ has the equivalent morphologies with that of the QSI of their canonical quadratic forms $\overline{\mathcal{A}}$ and $\overline{\mathcal{B}}$ with quadric form $\bar{A}$ and $\bar{B}$ given in Lemma 4 .

Note 3. The terminology "morphology" in Tu et al. (2009) takes topology, real and imaginary components, types and numbers of singularities into account. Our theoretical analysis on enumerations of morphologies of cyclides is based on Tu et al. (2009); however, in the final enumeration, we further distinguish the separability of the surface defined in Note 1, and we enumerate only the real component of the surface.

\subsubsection{Index sequences}

The characteristic polynomial of the pencil $\lambda A-B$ in $\mathbb{P R}^{4}$ is defined by

$$
f(\lambda)=\operatorname{det}(\lambda A-B),
$$


Table 1: The notions (the first column) represent a real root in place of $\uparrow$, depending on the size and sign of Jordan block (the second column) associated to the corresponding eigenvalue of $A^{-1} B$. The index jump value (the third column) across each real root depends on the Jordan form.

\begin{tabular}{|c|c|c|}
\hline notion & Jordan form & index jump \\
\hline & $1 \times 1$ Jordan block & +1 or -1 \\
\hline$\varkappa_{+}$or $\varkappa_{-}$ & $2 \times 2$ Jordan block with the sign + or - & 0 \\
\hline $222_{+}$or $222_{-}$ & $3 \times 3$ Jordan block with the sign + or - & +1 or -1 \\
\hline $2222_{+}$or $2222_{-}$ & $4 \times 4$ Jordan block with the $\operatorname{sign}+$ or - & 0 \\
\hline $22222_{+}$or $22222_{-}$ & $5 \times 5$ Jordan block with the sign + or - & +1 or -1 \\
\hline
\end{tabular}

which is a degree 5 polynomial with real coefficients. In order to guarantee the existence of nonsingular quadrics, we assume the characteristic polynomial dose not vanish identically.

Index function: The index function $\operatorname{Id}(\lambda)$ of a quadric pencil $\lambda A-B$ is defined as the number of positive eigenvalues of the matrix $\lambda A-B$. In our discussion, both matrices $A$ and $B$ are of order 5 , so $\operatorname{Id}(\lambda) \in\{0,1,2,3,4,5\}$. Since $\lambda A-B$ is symmetric, it represents a sphere when $\operatorname{rank}(\lambda A-B)=5$ and $\operatorname{Id}(\lambda)=4$. Note that $\operatorname{Id}(\lambda)$ has a constant value in the interval between any two consecutive real roots of $f(\lambda)=0$, and has a jump across a real root of $f(\lambda)=0$, whose jumping value depends on the Jordan block associated with the root as an eigenvalue of the matrix $\lambda A-B$, see the second and third columns in Table 1. Besides, $\operatorname{Id}(+\infty)+\operatorname{Id}(-\infty)=\operatorname{rank}(A)$ (Tu et al. 2009).

Index sequence: Let $\lambda_{i}, i=1, \ldots, r$ be all different real roots of $f(\lambda)=0$ in the ascending order. Let $q_{k}, k=1, \ldots r-1$ denote arbitrary rational numbers disconnected the $\lambda_{i}$, i.e.,

$$
-\infty<\lambda_{1}<q_{1}<\lambda_{2}<\ldots<q_{r-1}<\lambda_{r}<\infty .
$$

Denote $s_{k}=\operatorname{Id}\left(q_{k}\right), k=1,2, \ldots, r-1$, and $s_{0}=\operatorname{Id}(-\infty), s_{r}=\operatorname{Id}(\infty)$. Then the index sequence of $\mathcal{A}$ and $\mathcal{B}$ is defined as

$$
\left\langle s_{0} \uparrow s_{1} \uparrow \ldots \uparrow s_{r-1} \uparrow s_{r}\right\rangle,
$$

where $\uparrow$ stands for a real root, single or multiple, of $f(\lambda)=0$. Since a real root $\lambda_{i}$ can be multiple, and the Jordan chain associated with a multiple root $\lambda_{i}$ of the matrix $\lambda_{i} A-B$ can have distinct forms, we shall indicate these distinct forms in place of $\uparrow$ in the index sequence, see the first column in Table 1 . For example, a real root with Segre characteristic [11] will be denoted by $\|$ in place of an $\uparrow$, and a real root with the Segre characteristic [21] shall be denoted by $\imath_{+} \mid$or $\imath_{-} \mid$in place of an $\uparrow$. Examples of index sequences are: $\langle 1|2| 1|2| 3 \mid 4\rangle,\left\langle 1 \imath_{-} 1|2| \mid 4\right\rangle,\left\langle 2 \imath \imath_{+} 3 \imath_{+} 3\right\rangle$, etc.

Lemma 6. (Tu et al. 2009) The QSIs of different quadric pencils whose index sequences are in the same equivalent class under the following rules are of the same morphologies.

\section{(1) Rotation rule:}

$$
\begin{gathered}
<s_{0} \uparrow s_{1} \uparrow \ldots \uparrow s_{r-1} \uparrow s_{r}>\sim<5-s_{r-1} \uparrow s_{0} \uparrow \ldots \uparrow s_{r-2} \uparrow s_{r-1}>, \\
<s_{0} \uparrow s_{1} \uparrow \ldots \uparrow s_{r-1} \uparrow s_{r}>\sim<s_{1} \uparrow s_{2} \uparrow \ldots \uparrow s_{r} \uparrow 5-s_{1}>.
\end{gathered}
$$

(2) Reversal rule:

$$
<s_{0} \uparrow s_{1} \uparrow \ldots \uparrow s_{r-1} \uparrow s_{r}>\sim<s_{r} \uparrow s_{r-1} \uparrow \ldots \uparrow s_{1} \uparrow s_{0}>
$$

\section{(3) Complement rule:}

$$
<s_{0} \uparrow s_{1} \uparrow \ldots \uparrow s_{r-1} \uparrow s_{r}>\sim<5-s_{0} \uparrow 5-s_{1} \uparrow \ldots \uparrow 5-s_{r-1} \uparrow 5-s_{r}>.
$$


Table 2: The first column indicates the multiplicity of the real roots of the characteristic polynomial, the second and the third column are the corresponding Segre characteristic.

\begin{tabular}{|c|c|c|}
\hline real root & valid Segre characteristic & invalid Segre characteristic \\
\hline 5 simple & {$[11111]_{5}$} & $/$ \\
\hline 3 simple & {$[11111]_{3}$} & $/$ \\
\hline 1 simple & $/$ & {$[1111]_{1}$} \\
\hline 1 double & {$[2111]_{4}[(11) 111]_{4}[(11) 111]_{2}$} & {$[2111]_{2}$} \\
\hline 2 double & {$[21(11)]_{3}[(11)(11) 1]_{3}$} & {$[311]_{1}[(21) 11]_{1}$} \\
\hline 1 triple & {$[311]_{3}[(21) 11]_{3}[(111) 11]_{3}[(111) 11]_{1}$} & {$[32]_{2}[(21) 2]_{2}$} \\
\hline 1 triple +1 double & {$[3(11)]_{2}[(21)(11)]_{2}[(111)(11)]_{2}[(111) 2]_{2}$} & {$[41]_{2}[(22) 1]_{2}$} \\
\hline 1 quadruple & {$[(31) 1]_{2}[(211) 1]_{2}[(1111) 1]_{2}$} & {$[(311)]_{1}[(2111)]_{1}$} \\
\hline 1 quintuple & & {$[5]_{1}[(41)]_{1}[(32)]_{1}[(1111)]_{1}$} \\
\hline
\end{tabular}

\section{Darboux cyclides classification}

We now enumerate the morphologies of Darboux cyclides by investigating QSIs in $\mathbb{P R}^{4}$ by

1. Enumerate all valid Segre characteristics;

2. For each valid Segre characteristic, enumerate all valid and non-equivalent index sequences;

3. For each valid index sequence, enumerate the morphologies of the Darboux cyclide derived from the corresponding QSI;

4. Classify all enumerated Darboux cyclides by their morphologies.

\subsection{Validity of Segre characteristic/index sequence/QSI}

Definition 4.1. The signature of a quadric $X^{T} A X=0$ in $\mathbb{P R}^{4}$ is the integer pair $\left(n_{+}, n_{-}\right)$, where $n_{+}, n_{-}$are numbers of the positive eigenvalues and negative eigenvalues of the matrix $A$.

Definition 4.2. A Segre characteristic/index sequence/QSI is said to be valid, if there exists a quadric pencil $\lambda A-B$ in $\mathbb{P R}^{4}$ with this Segre characteristic/index sequence/QSI that has a quadric $\lambda^{*} A-B$ with the signature $(4,1)$ or $(1,4)$ for some constant $\lambda^{*} \in \mathbb{P R}^{1}$; otherwise we say the Segre characteristic/index sequence/QSI is invalid.

Proposition 4.1. An index sequence is valid if and only if it is equivalent under the rotation rule, reversal rule, complement rule to an index sequence which contains the index 1 or 4 . For example, $\left\langle 2 \imath_{+} 3|4| 3\right\rangle$ is valid; $\langle 2|3| 2|3| 2 \mid 3\rangle$ is invalid since it is non-equivalent to any index sequences containing the index 1 or 4 . A QSI in $\mathbb{P R}^{4}$ is valid if and only if its corresponding index sequence is valid. We say that a Segre characteristic is valid if there is at least one valid index sequence corresponding to it.

Since the characteristic polynomial $f(\lambda)=0$ is of degree 5, all Segre characteristics of quadric pencils in $\mathbb{P R}^{4}$ are enumerated in Table 2, with valid and invalid ones separately listed. The validity of the Segre characteristic can be checked by the validity of all their corresponding index sequences using Proposition 4.1 .

\subsection{Enumeration of valid index sequences and morphologies of Darboux Cyclides}

For each Segre characteristic, we enumerate all valid index sequences (step 2) as follows: 
(1) The index numbers in the sequence can only be from $\{0,1,2,3,4,5\}$;

(2) The index jump across each real root follows the law in Table 1

(3) Only a representative one is chosen for each equivalent class of the sequence under the rules in Lemma 6

(4) Check the validity of the representative index sequence.

Each valid index sequence corresponds to a QSI morphologies, from which we enumerate Darboux cyclides in the following way:

(i) Write down two canonical quadrics $\overline{\mathcal{A}}$ and $\overline{\mathcal{B}}$ in $\mathbb{P R}^{4}$;

(ii) Find two quadrics $\mathcal{A}^{*}$ and $\mathcal{B}^{*}$ in the pencil $\lambda \bar{A}-\bar{B}$ so that $\mathcal{A}^{*}$ has signature $(4,1)$ or $(1,4)$;

(iii) Enumerate four representative affine realizations of the QSI from $\mathbb{P R}^{4}$ to $\mathbb{R}^{4}$ (see Remark 4.1);

(iv) Compute stereographic projection of each QSI in $\mathbb{R}^{4}$ to get all Darboux cyclides.

Remark 4.1. Suppose the quadric $\mathcal{A}^{*}$ in step (ii) has signature $(4,1)$ and equation $X^{T} A^{*} X=0$, where $X=[x, y, z, u, v]^{T}$ and $A^{*}=\operatorname{diag}\left(\lambda_{1}, \lambda_{2}, \lambda_{3}, \lambda_{4}, \lambda_{5}\right)$. Without loss of generality, we assume that $\lambda_{i}>0, i=1,2,3,4$ and $\lambda_{5}<0$. On one hand, letting $v=0$ be the plane at infinity realizes an affine sphere in $\mathbb{R}^{4}$; on the other hand, there are four ways of realizing the other four axes in the follow-up stereographic projection in Equation (2): letting $x=x_{4}, y=x_{4}, z=x_{4}$ or $u=x_{4}$. Note that since the projection center is $(0,0,0,1)$, the axes $x_{1}, x_{2}, x_{3}$ have symmetric performance and only the assignment of $x_{4}$ matters. Therefore, for each QSI in $\mathbb{P R}^{4}$, we enumerate all these four affine realizations of $\mathbb{R}^{4}$ and the derived Darboux cyclides.

We next show the above process in every detail for the Segre characteristic [11111 $]_{5}$.

Theorem 2. Given two quadrics $\mathcal{A}: X^{T} A X=0$ and $\mathcal{B}: X^{T} B X=0$, if the Segre characteristic of the quadric pencil is $[11111]_{5}$, then the pencil has the following valid index sequences and corresponding morphologies of Darboux cyclides:

(1) $\langle 1|2| 1|2| 3 \mid 4\rangle$

(a) Case 1.1: topologically two spheres, with one inside the other, no singularities;

(b) Case 1.2: topologically two separate spheres, no singularities.

(2) $\langle 1|2| 3|2| 3 \mid 4\rangle$

(a) Case 2: topologically a torus, no singularities.

Proof. The Segre characteristic suggests that $f(\lambda)=0$ has five distinct real roots $\lambda_{1}<\lambda_{2}<\lambda_{3}<$ $\lambda_{4}<\lambda_{5}$. According to Lemma 4 , the quadric pair canonical form is

$$
\bar{A}=\operatorname{diag}\left(\varepsilon_{1}, \varepsilon_{2}, \varepsilon_{3}, \varepsilon_{4}, \varepsilon_{5}\right) \text { and } \bar{B}=\operatorname{diag}\left(\varepsilon_{1} \lambda_{1}, \varepsilon_{2} \lambda_{2}, \varepsilon_{3} \lambda_{3}, \varepsilon_{4} \lambda_{4}, \varepsilon_{5} \lambda_{5}\right),
$$

where $\varepsilon_{i}= \pm 1, i=1,2,3,4,5$. Since $\operatorname{Id}(-\infty)+\operatorname{Id}(+\infty)=5$, the index sequence must have the form $\langle 0|*| *|*| * \mid 5\rangle,\langle 1|*| *|*| * \mid 4\rangle$ or $\langle 2|*| *|*| * \mid 3\rangle$. Considering the equivalent rules in Lemma6 6 and that the index jump across each simple root is \pm 1 , all representative index sequences are $\langle 0|1| 2|3| 4 \mid 5\rangle$, $\langle 1|2| 1|2| 3 \mid 4\rangle,\langle 1|2| 3|2| 3 \mid 4\rangle$ and $\langle 2|3| 2|3| 2 \mid 3\rangle$. 
(1) If the index sequence is $\langle 0|1| 2|3| 4 \mid 5\rangle$, then $\varepsilon_{1}=\varepsilon_{2}=\varepsilon_{3}=\varepsilon_{4}=\varepsilon_{5}=1$, i.e.,

$$
\bar{A}=\operatorname{diag}(1,1,1,1,1) \text { and } \bar{B}=\operatorname{diag}\left(\lambda_{1}, \lambda_{2}, \lambda_{3}, \lambda_{4}, \lambda_{5}\right) .
$$

the corresponding canonical quadrics are

$$
x^{2}+y^{2}+z^{2}+u^{2}+v^{2}=0 \text { and } \lambda_{1} x^{2}+\lambda_{2} y^{2}+\lambda_{3} z^{2}+\lambda_{4} u^{2}+\lambda_{5} v^{2}=0 .
$$

Since $\bar{A}$ is a vacuous sphere, the QSI is also vacuous, we omit this case in Table 5 .

(2) If the index sequence is $\langle 1|2| 1|2| 3 \mid 4\rangle$, then $\varepsilon_{1}=1, \varepsilon_{2}=-1, \varepsilon_{3}=\varepsilon_{4}=\varepsilon_{5}=1$, i.e,

$$
\bar{A}=\operatorname{diag}(1,-1,1,1,1) \text { and } \bar{B}=\operatorname{diag}\left(\lambda_{1},-\lambda_{2}, \lambda_{3}, \lambda_{4}, \lambda_{5}\right),
$$

the corresponding canonical quadrics are:

$$
x^{2}-y^{2}+z^{2}+u^{2}+v^{2}=0 \text { and } \lambda_{1} x^{2}-\lambda_{2} y^{2}+\lambda_{3} z^{2}+\lambda_{4} u^{2}+\lambda_{5} v^{2}=0 .
$$

Letting $y=0$ be the plane at infinity, we have a sphere and a quadric in $\mathbb{R}^{4}$ :

$$
\overline{\mathcal{A}}: x^{2}+z^{2}+u^{2}+v^{2}=1 \text { and } \overline{\mathcal{B}}: \lambda_{1} x^{2}+\lambda_{3} z^{2}+\lambda_{4} u^{2}+\lambda_{5} v^{2}-\lambda_{2}=0 .
$$

Letting $x_{1}=z, x_{2}=u, x_{3}=v, x_{4}=x$, the stereographic projection in Equation (2) through center $(0,0,0,1)$ gives

$D_{1}=\left(\lambda_{2}-\lambda_{1}\right)\left(x^{2}+y^{2}+z^{2}\right)^{2}+2\left(\lambda_{1}+\lambda_{2}\right)\left(x^{2}+y^{2}+z^{2}\right)-4\left(\lambda_{3} x^{2}+\lambda_{4} y^{2}+\lambda_{5} z^{2}\right)+\lambda_{2}-\lambda_{1}=0$,

whose morphology is topologically two spheres, with one inside the other, no singularities, denoted as Case 1.1 in Table 5 similarly, we can prove that the other three ways of realizing $\mathbb{R}^{4}$ by setting $x_{4}=y$ or $x_{4}=u$ or $x_{4}=v$ give the same Darboux cyclide, which is topologically two separate spheres, no singularities, denoted by Case 1.2 in Table 5

(3) If the index sequence is $\langle 1|2| 3|2| 3 \mid 4\rangle$, then $\varepsilon_{1}=\varepsilon_{2}=1, \varepsilon_{3}=-1, \varepsilon_{4}=\varepsilon_{5}=1$, i.e,

$$
\bar{A}=\operatorname{diag}(1,1,-1,1,1) \text { and } \bar{B}=\operatorname{diag}\left(\lambda_{1}, \lambda_{2},-\lambda_{3}, \lambda_{4}, \lambda_{5}\right) .
$$

Choosing $z=0$ as the plane at infinity realizes a sphere and a quadric in $\mathbb{R}^{4}$ :

$$
\overline{\mathcal{A}}: x^{2}+y^{2}+u^{2}+v^{2}=1 \text { and } \overline{\mathcal{B}}: \lambda_{1} x^{2}+\lambda_{2} y^{2}+\lambda_{4} u^{2}+\lambda_{5} v^{2}-\lambda_{3}=0 .
$$

Letting $x_{4}=x, x_{1}=y, x_{2}=u, x_{3}=v$ gives a realization of $\mathbb{R}^{4}$. The stereographic projection through center $\mathbf{O}=(0,0,0,1)$ gives

$D_{2}=\left(\lambda_{5}-\lambda_{3}\right)\left(x^{2}+y^{2}+z^{2}\right)^{2}-2\left(\lambda_{5}+\lambda_{3}\right)\left(x^{2}+y^{2}+z^{2}\right)+4\left(\lambda_{1} x^{2}+\lambda_{2} y^{2}+\lambda_{4} z^{2}\right)+\lambda_{5}-\lambda_{3}=0$,

which is a ring cyclide; similarly, we can prove that the other three ways of realizing $\mathbb{R}^{4}$ by setting $x_{4}=y$ or $x_{4}=u$ or $x_{4}=v$ give the same Darboux cyclide morphologies, denoted by Case 2 in Table 5 .

(4) If the index sequence is $\langle 2|3| 2|3| 2 \mid 3\rangle$, from Proposition 4.1 , we know this index sequence is invalid. 
Index sequences and morphologies of Darboux cyclides for other Segre characteristics can be similarly analyzed. Readers are referred to the technical report for detailed proofs. Table 547 show all the enumeration results: the first column is the Segre characteristic with the subscript indicating the number of real roots, not counting multiplicities; the second column is all the valid index sequences under each Segre characteristic; the third column gives a example of a pair of quadrics (one of which is an affine sphere in $\mathbb{R}^{4}$ ) in the quadric pencil in $\mathbb{P R}^{4}$; and the fourth and fifth columns show the morphology and equation of the Darboux cyclide computed from the stereographic projection of the QSIs. Here, a solid dot indicates a real acnode. Note that in Table 5 7, we have excluded the degenerate Darboux cyclides, however, all such exclusions will be addressed in the appendix.

Note that there can be more than one appearance for a derived Darboux cyclide, depending on the coefficient sign of the quartic term. For example, for the index sequence $\left\langle 1\left|2 \imath_{+} 2\right| 3 \mid 4\right\rangle$, one derived equation of the Darboux cyclide (see details in the appendix) is

$$
D=\left(\lambda_{1}-\lambda_{2}+\frac{1}{2}\right) \mathbf{X}^{2}-2\left(\lambda_{1}+\lambda_{2}-x+\frac{1}{2}\right) \mathbf{X}+4\left(\lambda_{2} x^{2}+\lambda_{3} y^{2}+\lambda_{4} z^{2}\right)+2 x^{2}+2 x+\lambda_{1}-\lambda_{2}+\frac{1}{2}=0,
$$

where $\mathbf{X}=x^{2}+y^{2}+z^{2}$ and $\lambda_{1}<\lambda_{2}<\lambda_{3}<\lambda_{4}$ are the four real roots of the characteristic polynomial $f(\lambda)=0$. If $\lambda_{1}-\lambda_{2}+\frac{1}{2}<0$, the morphology of this Darboux cyclide is Case 6.1 , shown in Table 5 . if $\lambda_{1}-\lambda_{2}+\frac{1}{2}>0$, the morphology is Case $6.1^{*}$, also shown in Table 5 if $\lambda_{1}-\lambda_{2}+\frac{1}{2}=0$ the cyclide degenerates to a cubic surface with a singular point, which is beyond our investigation scope. The cause of this phenomenon is from different relative positions of the projection center with respect to the QSI in $\mathbb{R}^{4}$. In geometry, these two morphologies are essentially the same. Nevertheless, we still distinguish them to provide better understanding of 3D shapes of Darboux cyclides.

\subsubsection{Dupin cyclides}

We further address which Darboux cyclides in Table 50 are Dupin cyclides. In Table 5 77, we add an upper right subscript in the fourth column of Illustration to indicate whether the Darboux cyclide is a Dupin cyclide. ' $\mathrm{H}, \mathrm{R}, \mathrm{S}$ ' refer to horn Dupin cyclide, ring Dupin cyclide and spindle Dupin cyclide, respectively. We add 'Dupin' to distinguish them from the general Darboux cyclides.

The implicit equation of a Dupin cyclide is

$$
\left(x^{2}+y^{2}+z^{2}+b^{2}-d^{2}\right)^{2}-4(a x-c d)^{2}-4 b^{2} y^{2}=0,
$$

where $a>b>0, d \geq 0$, and $c^{2}=a^{2}-b^{2}$. The value of parameter $d$ classifies Dupin cyclides into horn cyclide, ring cyclide and spindle cyclide. Without loss of generality, we let $a=5, b=4$ and $c=3$.

(1) $d=0$, which corresponds to a symmetric horn cyclide. An example is

$$
\left(x^{2}+y^{2}+z^{2}+16\right)^{2}-100 x^{2}-64 y^{2} .
$$

By the inverse stereographic projection, i.e., Equation (3), we get

$$
100 x_{1}^{2}+64 x_{2}^{2}-225 x_{4}^{2}+510 x_{4}-289=0,
$$

which is a quadric in $\mathbb{R}^{4}$. The index sequence of the QSI obtained by this quadric and a sphere is $\langle 1|| 3|| 3 \mid 4\rangle$, which is Case 15.1 in Table 6, denoted by superscript $\mathrm{H}$.

(2) $0<d<c$, which corresponds to a non-symmetric horn cyclide. By setting $d=1$, an example is

$$
\left(x^{2}+y^{2}+z^{2}+15\right)^{2}-4(5 x-3)^{2}-64 y^{2}=0 .
$$


By the inverse stereographic projection, we get

$$
25 x_{1}^{2}+16 x_{2}^{2}-40 x_{4}^{2}+30 x_{1} x_{4}-30 x_{1}+94 x_{4}-55=0,
$$

which is a quadric in $\mathbb{R}^{4}$. The index sequence of the QSI obtained by this quadric and a sphere is $\langle 1|| 3|| 3 \mid 4\rangle$, which is Case 15.1 in Table 6, denoted by superscript $\mathrm{H}$.

(3) $d=c$, which corresponds to a horn cyclide, whose morphology is different from the first two cases. An example is

$$
\left(x^{2}+y^{2}+z^{2}+7\right)^{2}-4(5 x-9)^{2}-64 y^{2}=0 .
$$

By the inverse stereographic projection, we get

$$
25 x_{1}^{2}+16 x_{2}^{2}+72 x_{4}^{2}+90 x_{1} x_{4}-90 x_{1}-138 x_{4}+65=0,
$$

which is a quadric in $\mathbb{R}^{4}$. The index sequence of the QSI obtained by this quadric and a sphere is $\left\langle 1|| 3 \imath_{+} \mid 4\right\rangle$, which is Case 21.1 in Table 7 , denoted by superscript $\mathrm{H}$.

(4) $c<d$, which corresponds to a ring cyclide. By setting $d=4$, an example is

$$
\left(x^{2}+y^{2}+z^{2}\right)^{2}-4(5 x-12)^{2}-64 y^{2}=0 .
$$

By the inverse stereographic projection, we get

$$
100 x_{1}^{2}+64 x_{2}^{2}+575 x_{4}^{2}+480 x_{1} x_{4}-480 x_{1}-1154 x_{4}+575=0,
$$

which is a quadric in $\mathbb{R}^{4}$. The index sequence of the QSI obtained by this quadric and a sphere is $\langle 1|| 3|2| \mid 4\rangle$, which is Case 14 in Table 6, denoted by superscript $\mathrm{R}$.

(5) $d=a$, which corresponds to a ring cyclide, whose morphology is distinct to Case 14. An example is

$$
\left(x^{2}+y^{2}+z^{2}-9\right)^{2}-4(5 x-15)^{2}-64 y^{2}=0 .
$$

By the inverse stereographic projection, we get

$$
25 x_{1}^{2}+16 x_{2}^{2}+200 x_{4}^{2}+150 x_{1} x_{4}-150 x_{1}-410 x_{4}+209=0,
$$

which is a quadric in $\mathbb{R}^{4}$. The index sequence of the QSI obtained by this quadric and a sphere is $\left\langle 1|| 3 \imath_{+} \mid 4\right\rangle$, which is Case 21.2 in Table 7 , denoted by superscript $\mathrm{R}$.

(6) $a<d$, which corresponds to a spindle cyclide. By setting $d=8$, an example is

$$
\left(x^{2}+y^{2}+z^{2}-48\right)^{2}-4(5 x-24)^{2}-64 y^{2}=0 .
$$

By the inverse stereographic projection, we get

$$
100 x_{1}^{2}+960 x_{1} x_{4}+64 x_{2}^{2}-97 x_{4}^{2}-960 x_{1}-2 x_{4}+95=0,
$$

which is a quadric in $\mathbb{R}^{4}$. The index sequence of the QSI obtained by this quadric and a sphere is $\langle 1|| 3|| 3 \mid 4\rangle$, which is Case 15.2 in Table 6 , denoted by superscript $\mathrm{S}$.

\subsection{Morphology classification of Darboux cyclides}

The previous enumeration is based on the algebraic equivalence of index sequences and Segre characteristics of a sphere and a quadric in $\mathbb{P R}^{4}$. It is still possible that some of these enumerated index sequences in Table 57 correspond to an equivalent morphology under our classification rules in Section 3. For instance, Case 1.1 and Case 8.2 in Table 5 have the equivalent morphology, which is topologically two spheres, with one inside the other, no singularities, denoted by $M_{1}$ in Table 3 . Table 3 shows our final classification result with a total number of 14 non-equivalent morphologies. The third column of Table 3 shows the index sequences from Table 57 that give the corresponding morphologies. Table 4 provides every detail on the topology, singularity number and type as well as separability of the 14 non-equivalent morphologies in Table 3. 
Table 3: Classifications of non-equivalent morphologies of Darboux cyclides. The second and fifth columns show the case IDs from the upper-left corner of the fourth columns in Table 55 7. The third and sixth columns show the corresponding index sequence for each case.

\begin{tabular}{|c|c|c|c|c|c|}
\hline $\operatorname{Morp}_{i}$ & $\mathbf{I D}_{j}$ & Index Sequence & $\operatorname{Morp}_{i}$ & $\mathbf{I D}_{j}$ & Index Sequence \\
\hline \multirow{2}{*}{$M_{1}$} & $\mathrm{ID}_{1.1}$ & $\langle 1|2| 1|2| 3 \mid 4\rangle$ & \multirow{3}{*}{$M_{8}$} & $\mathrm{ID}_{18.2}$ & $\left\langle 1 \varkappa_{-}|2| 3 \mid 4\right\rangle$ \\
\hline & $\mathrm{ID}_{8.2}$ & $\langle 1|| 3|4| 3 \mid 4\rangle$ & & $\mathrm{ID}_{21.1}$ & $\left\langle 1|| 3 \imath_{+} \mid 4\right\rangle$ \\
\hline \multirow{2}{*}{$M_{2}$} & $\mathrm{ID}_{1.2}$ & $\langle 1|2| 1|2| 3 \mid 4\rangle$ & & $\mathrm{ID}_{17}$ & $\left\langle 1\left|2 \imath \iota \iota_{+} 3\right| 4\right\rangle$ \\
\hline & $\mathrm{ID}_{8.1}$ & $\langle 1|| 3|4| 3 \mid 4\rangle$ & \multirow{3}{*}{$M_{9}$} & $\mathrm{ID}_{6.1}$ & $\left\langle 1\left|2 \imath_{+} 2\right| 3 \mid 4\right\rangle$ \\
\hline \multirow{3}{*}{$M_{3}$} & $\mathrm{ID}_{2}$ & $\langle 1|2| 3|2| 3 \mid 4\rangle$ & & $\mathrm{ID}_{13.1}$ & $\left\langle 1\left|2 \imath_{+} 2\right| \mid 4\right\rangle$ \\
\hline & $\mathrm{ID}_{7}$ & $\langle 1|| 3|2| 3 \mid 4\rangle$ & & ID $_{19.1}$ & $\left\langle 1\left|2 \imath_{+}\right| 3 \mid 4\right\rangle$ \\
\hline & $\mathrm{ID}_{14}$ & $\langle 1|| 3|2| \mid 4\rangle$ & \multirow{3}{*}{$M_{10}$} & $\mathrm{ID}_{6.1^{*}}$ & $\left\langle 1\left|2 \imath_{+} 2\right| 3 \mid 4\right\rangle$ \\
\hline \multirow{2}{*}{$M_{4}$} & $\mathrm{ID}_{3}$ & $\langle 1|2| 3 \mid 4\rangle$ & & $\mathrm{ID}_{13.1 *}$ & $\left\langle 1\left|2 \imath_{+} 2\right| \mid 4\right\rangle$ \\
\hline & $\mathrm{ID}_{10}$ & $\langle 1|| 3 \mid 4\rangle$ & & $\mathrm{ID}_{19.1 *}$ & $\left\langle 1\left|2 \imath_{+}\right| 3 \mid 4\right\rangle$ \\
\hline \multirow{2}{*}{$M_{5}$} & $\mathrm{ID}_{4.1}$ & $\left\langle 1 \chi_{-} 1|2| 3 \mid 4\right\rangle$ & \multirow{2}{*}{$M_{11}$} & $\mathrm{ID}_{9.1}$ & $\langle 1|2||2| 3 \mid 4\rangle$ \\
\hline & $\mathrm{ID}_{11.1}$ & $\left\langle 1 \varkappa_{-} 1|2| \mid 4\right\rangle$ & & $\mathrm{ID}_{15.2}$ & $\langle 1|| 3|| 3 \mid 4\rangle$ \\
\hline \multirow{2}{*}{$M_{6}$} & $\mathrm{ID}_{4.2}$ & $\left\langle 1 u_{-} 1|2| 3 \mid 4\right\rangle$ & \multirow{2}{*}{$M_{12}$} & $\mathrm{ID}_{9.2}$ & $\langle 1|2||2| 3 \mid 4\rangle$ \\
\hline & $\mathrm{ID}_{11.2}$ & $\left\langle 1 \varkappa_{-} 1|2| \mid 4\right\rangle$ & & $\mathrm{ID}_{15.1}$ & $\langle 1|| 3|| 3 \mid 4\rangle$ \\
\hline \multirow{4}{*}{$M_{7}$} & $\mathrm{ID}_{5.1}$ & $\left\langle 1\left|2 \varkappa_{-} 2\right| 3 \mid 4\right\rangle$ & \multirow{3}{*}{$M_{13}$} & $\mathrm{ID}_{16.1}$ & $\langle 12222+2|3| 4\rangle$ \\
\hline & $\mathrm{ID}_{12.1}$ & $\left\langle 1\left|2 \imath_{-} 2\right| \mid 4\right\rangle$ & & $\mathrm{ID}_{20.1}$ & $\langle 122 \iota+2 \| 4\rangle$ \\
\hline & $\mathrm{ID}_{18.1}$ & $\left\langle 1 \varkappa_{-}|2| 3 \mid 4\right\rangle$ & & $\mathrm{ID}_{22.1}$ & $\langle 1222+|3| 4\rangle$ \\
\hline & $\mathrm{ID}_{21.2}$ & $\left\langle 1|| 3 \imath_{+} \mid 4\right\rangle$ & \multirow{3}{*}{$M_{14}$} & $\mathrm{ID}_{16.2}$ & $\langle 1 \prec 2 \iota+2|3| 4\rangle$ \\
\hline \multirow{2}{*}{$M_{8}$} & $\mathrm{ID}_{5.2}$ & $\left\langle 1\left|2 \chi_{-} 2\right| 3 \mid 4\right\rangle$ & & $\mathrm{ID}_{20.2}$ & $\langle 1222+2 \| 4\rangle$ \\
\hline & $\mathrm{ID}_{12.2}$ & $\left\langle 1\left|2 \chi_{-} 2\right| \mid 4\right\rangle$ & & $\mathrm{ID}_{22.2}$ & $\left\langle 1 \imath 2 \imath_{+}|3| 4\right\rangle$ \\
\hline
\end{tabular}


Table 4: Descriptions of the topology, singularity (number and type), and separability of the 14 non-equivalent morphologies in Table 3

\begin{tabular}{|c|c|c|c|c|c|c|c|c|c|}
\hline \multirow{2}{*}{ Morphology } & \multirow{2}{*}{ Illustration } & \multicolumn{2}{|c|}{ Singularity } & \multirow{2}{*}{ Separability } & \multirow{2}{*}{ Morphology } & \multirow{2}{*}{ Illustration } & \multicolumn{2}{|c|}{ Singularity } & \multirow{2}{*}{ Separability } \\
\hline & & Num & Type & & & & Num & Type & \\
\hline$M_{1}$ & & 0 & 1 & $\mathrm{~N}$ & $M_{8}$ & & 1 & crunode & $\mathrm{N}$ \\
\hline$M_{2}$ & & 0 & $\backslash$ & $\mathrm{Y}$ & $M_{9}$ & & 1 & crunode & $\mathrm{N}$ \\
\hline$M_{3}$ & & 0 & $\backslash$ & $\mathrm{N}$ & $M_{10}$ & & 1 & crunode & Y \\
\hline$M_{4}$ & & 0 & $\backslash$ & $\mathrm{N}$ & $M_{11}$ & & 2 & crunode & $\mathrm{N}$ \\
\hline$M_{5}$ & - & 1 & acnode & $\mathrm{N}$ & $M_{12}$ & & 2 & crunode & Y \\
\hline$M_{6}$ & - & 1 & acnode & Y & $M_{13}$ & & 1 & cusp & $\mathrm{N}$ \\
\hline$M_{7}$ & 7 & 1 & crunode & $\mathrm{N}$ & $M_{14}$ & & 1 & cusp & $\mathrm{N}$ \\
\hline
\end{tabular}

\section{Conclusion}

We provide an enumeration of all possible morphologies of Darboux cyclides based on the result by Coolidge (1916) that every Darboux cyclide is the stereographic projection of the intersection (QSI) of a quadric and a sphere in $\mathbb{R}^{4}$. We first enumerate all valid QSIs in $\mathbb{P R}^{4}$ by two algebraic sequences: Segre characteristics and index sequences of the quadric pencil, which is a generalization of Tu et al. (2009) from $\mathbb{P R}^{3}$ to $\mathbb{P R}^{4}$. Then we go through every valid affine realization of the QSI from $\mathbb{P R}^{4}$ to $\mathbb{R}^{4}$, and use stereographic projection to achieve a full enumeration of the morphologies of Darboux cyclides. We have also pointed out which of the enumerated morphologies are essentially Dupin cyclides.

\section{Acknowledgements}

We thank Prof. Jerome Hoffmann from Louisiana State University for his helpful insights and suggestions. This work is supported by the NSF of China grant 61872354, the open funding project of State Key Laboratory of Virtual Reality Technology and Systems, Beihang University (Grant No.BUAAVR-17KF-06) and Open Projects Program of National Laboratory of Pattern Recognition (NLPR) (201700020).

\section{References}

Allen, S., Dutta, D., 1997. Cyclides in pure blending i. Computer Aided Geometric Design 14 (1), $51-75$.

Belmans, P., 2016. Segre symbols.

Blum, R., 1980. Circles on surfaces in the euclidean 3-space. Lecture Notes in Math 792, 213-221.

Bo, P., Pottmann, H., Kilian, M., Wang, W., Wallner, J., 2011. Circular arc structures. Vol. 30. ACM. 
Bromwich, T. J. I., 1906. Quadratic Forms and Their Classification by Means of Invariant-factors, by TJ I'A. Bromwich. Vol. 3. Cambridge University Press.

Casey, J., 1870. On cyclides and sphero-quartics. Philosophical Transactions of the Royal Society $161(0), 585-721$.

Cayley, A., 1873. On the cyclide. Quart. J. of Pure and Appl. Math 12, 148-165.

Chandru, V., Dutta, D., Hoffmann, C. M., 1989. On variable radius blending using dupin cyclides.

Coolidge, J., 1916. A treatise on the circle and the sphere. clarendon.

Darboux, G., 1880. Sur le contact des courbes et des surfaces. Bulletin des Sciences Mathématiques et Astronomiques 4 (1), 348-384.

Druoton, L., Langevin, R., Garnier, L., 2014. Blending canal surfaces along given circles using dupin cyclides. International Journal of Computer Mathematics 91 (3), 641-660.

Dupin, C., 1822. Applications de géométrie et de méchanique, a la marine, aux ponts et chaussées, etc., pour faire suite aux Développements de géométrie, par Charles Dupin.. Bachelier, successeur de Mme. Ve. Courcier, libraire.

Dupont, L., Lazard, D., Lazard, S., Petitjean, S., 2003. Near-optimal parameterization of the intersection of quadrics. In: Proceedings of the nineteenth annual symposium on Computational geometry. ACM, pp. 246-255.

Dupont, L., Lazard, D., Lazard, S., Petitjean, S., 2008a. Near-optimal parameterization of the intersection of quadrics: I. the generic algorithm. Journal of Symbolic Computation 43 (3), 168-191.

Dupont, L., Lazard, D., Lazard, S., Petitjean, S., 2008b. Near-optimal parameterization of the intersection of quadrics: Ii. a classification of pencils. Journal of Symbolic Computation 43 (3), 192-215.

Dupont, L., Lazard, D., Lazard, S., Petitjean, S., 2008c. Near-optimal parameterization of the intersection of quadrics: Iii. parameterizing singular intersections. Journal of Symbolic Computation $43(3), 216-232$.

Foufou, S., Garnier, L., 2004. Dupin cyclide blends between quadric surfaces for shape modeling. In: Computer Graphics Forum. Vol. 23. Wiley Online Library, pp. 321-330.

Foufou, S., Garnier, L., Pratt, M. J., 2005. Conversion of dupin cyclide patches into rational biquadratic bézier form. Lecture notes in computer science 3604, 201-218.

Franquiz, J., Paluszny, M., Tovar, F., 2006. Cyclides and the guiding circle. Mathematics and Computers in Simulation 73 (1), 168-174.

Jia, X., Wang, W., Choi, Y. K., Mourrain, B., Tu, C., 2016. Continuous detection of the variations of the intersection curve of two moving quadrics in 3-dimensional projective space. Journal of Symbolic Computation 73 (C), 221-243.

Krasauskas, R., Zubè, S., 2014. Rational bézier formulas with quaternion and clifford algebra weights. In: SAGA-Advances in ShApes, Geometry, and Algebra. Springer, pp. 147-166.

Kummer, E. E., 1865. Ueber die flächen vierten grades, auf welchen schaaren von kegelschnitten liegen. Journal für die reine und angewandte Mathematik 64, 66-76.

Lazard, S., Peñaranda, L. M., Petitjean, S., 2006. Intersecting quadrics: An efficient and exact implementation. Computational Geometry 35 (1-2), 74-99. 
Levin, J., 1976. A parametric algorithm for drawing pictures of solid objects composed of quadric surfaces. Communications of the ACM 19 (10), 555-563.

Levin, J. Z., 1979. Mathematical models for determining the intersections of quadric surfaces. Computer Graphics and Image Processing 11 (1), 73-87.

Lubbes, N., 2013. Families of circles on surfaces. Mathematics.

Martin, R. R., et al., 1982. Prinicpal patches for computational geometry. Ph.D. thesis, University of Cambridge.

Maxwell, A., 1868. On the cyclide. Quart. J. of Pure and Appl. Math 9, 111-126.

McLean, D., 1985. A method of generating surfaces as a composite of cyclide patches. The Computer Journal 28 (4), 433-438.

Miller, J. R., Goldman, R. N., 1995. Geometric algorithms for detecting and calculating all conic sections in the intersection of any 2 natural quadric surfaces. Graphical Models and Image Processing $57(1), 55-66$.

Mourrain, B., Técourt, J.-P., Teillaud, M., 2005. On the computation of an arrangement of quadrics in 3d. Computational Geometry 30 (2), 145-164.

Pottmann, H., Asperl, A., Hofer, M., Kilian, A., Bentley, D., 2007. Architectural geometry. Vol. 724. Bentley Institute Press Exton.

Pottmann, H., Schiftner, A., Bo, P., Schmiedhofer, H., Wang, W., Baldassini, N., Wallner, J., 2008. Freeform surfaces from single curved panels. ACM Transactions on Graphics (TOG) 27 (3), 76.

Pottmann, H., Shi, L., Skopenkov, M., 2012. Darboux cyclides and webs from circles. Computer Aided Geometric Design 29 (1), 77-97.

Pratt, M. J., 1990. Cyclides in computer aided geometric design. Computer Aided Geometric Design 7 (1), 221-242.

Pratt, M. J., 1995. Cyclides in computer aided geometric design ii. Computer Aided Geometric Design $12(2), 131-152$.

Segre, C., 1884. Studio sulle quadriche in uno spazio lineare ad un numero qualunque di dimensioni: memoria. E. Loescher.

Takeuchi, N., et al., 2000. Cyclides. Hokkaido Mathematical Journal 29 (1), 119-148.

Tu, C., Wang, W., Mourrain, B., Wang, J., 2009. Using signature sequences to classify intersection curves of two quadrics. Computer Aided Geometric Design 26 (3), 317-335.

Tu, C., Wang, W., Wang, J., 2002. Classifying the nonsingular intersection curve of two quadric surfaces. In: Geometric Modeling and Processing, 2002. Proceedings. IEEE, pp. 23-32.

Uhlig, F., 1976. A canonical form for a pair of real symmetric matrices that generate a nonsingular pencil. Linear Algebra and its Applications 14 (3), 189 - 209. URL http://www.sciencedirect.com/science/article/pii/0024379576900665

Wang, W., Goldman, R., Tu, C., 2003. Enhancing levin's method for computing quadric-surface intersections. Computer Aided Geometric Design 20 (7), 401-422.

Wang, W., Joe, B., Goldman, R., 2002. Computing quadric surface intersections based on an analysis of plane cubic curves. Graphical Models 64 (6), 335-367.

Wilf, I., Manor, Y., 1993. Quadric-surface intersection curves: shape and structure. Computer-Aided Design 25 (10), 633-643. 
Table 5: Enumeration of the morphologies of Darboux cyclides

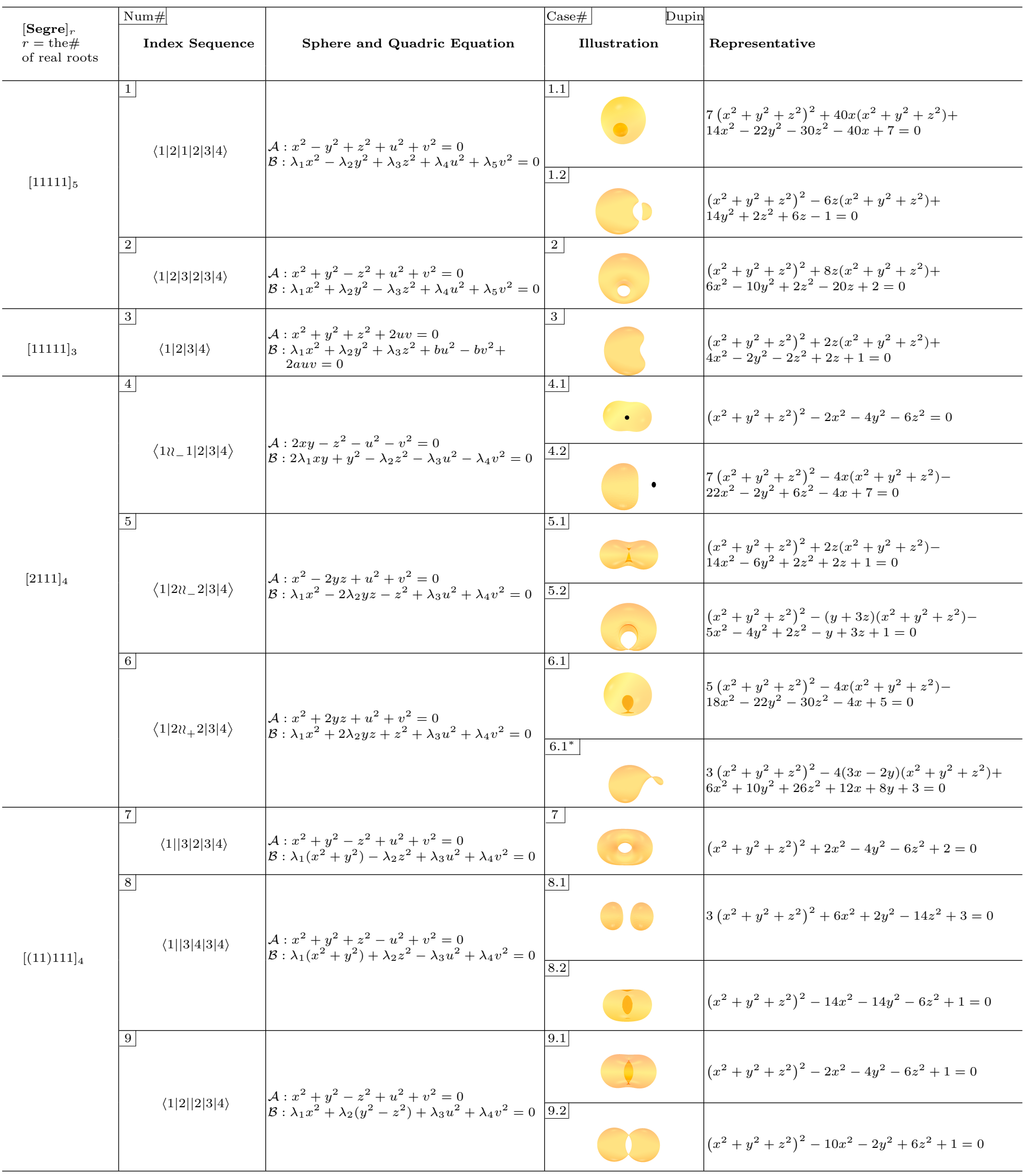


Table 6: Enumeration of the morphologies of Darboux cyclides

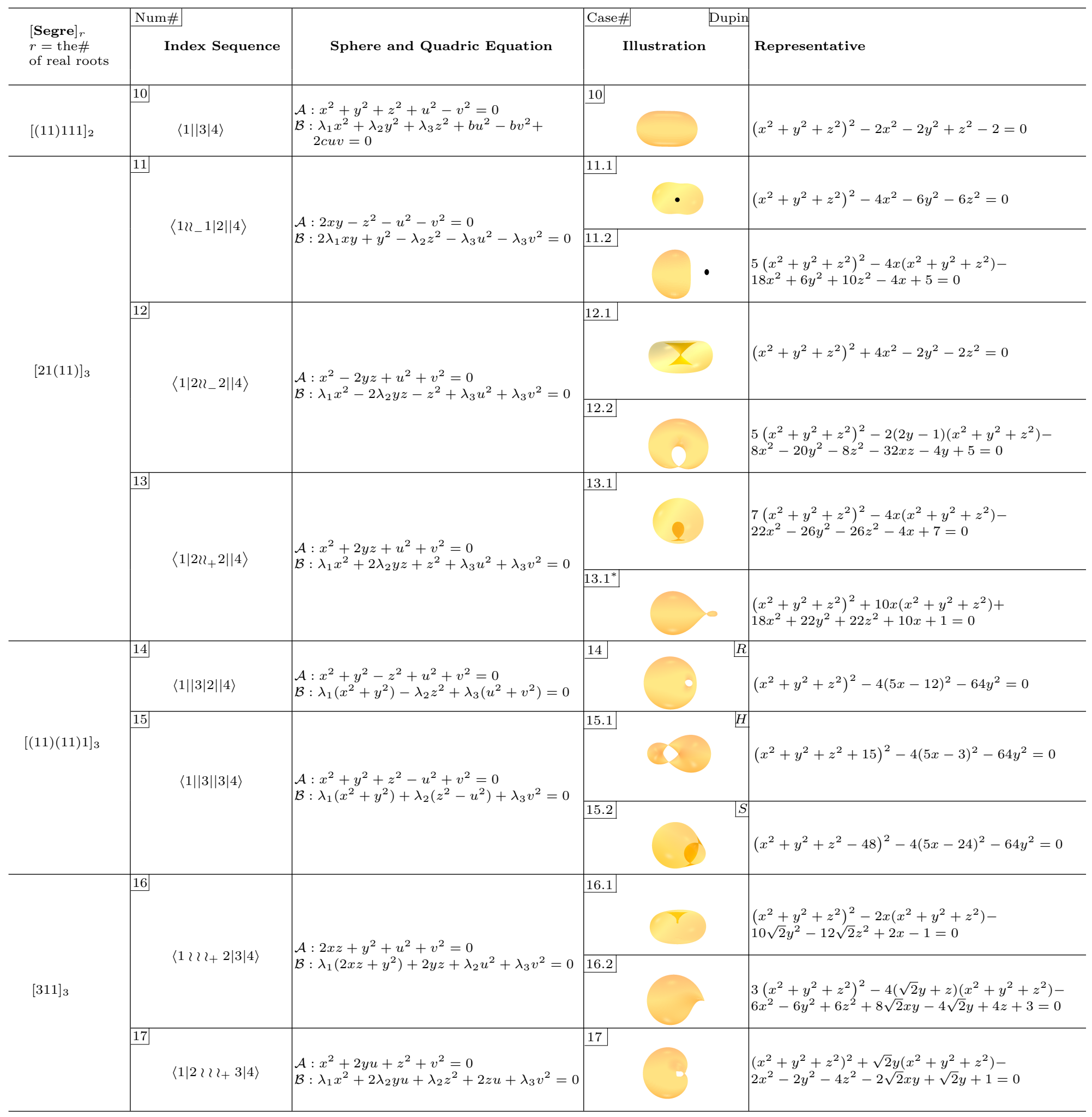


Table 7: Enumeration of the morphologies of Darboux cyclides

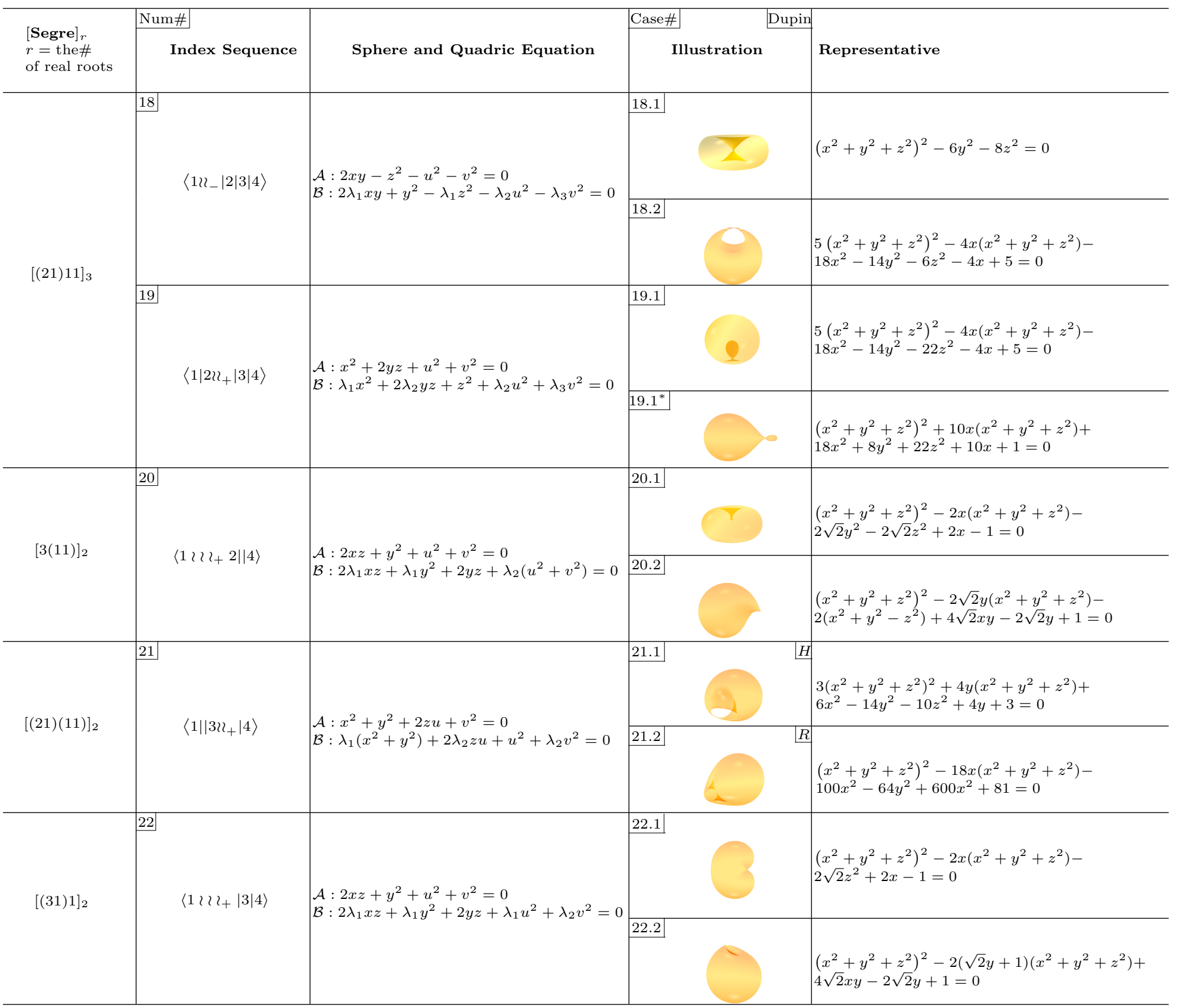

